\title{
Improving Data Spillage in Multi Cloud Capacity Administrations
}

\author{
M. Gowthami, S. Thanga Ramya
}

\begin{abstract}
Multi copy Dynamic information possession in distributed computing be able to to put missing information in dynamic methodology to the cloud server. Multi copy means, information to be imitated in different servers. The undertaking proprietor transfers the information into cloud server and the information is duplicated, then thatphotocopies are place away in several servers. The information is stored in multiple servers in order to avoid data loss due to vulnerabilities like hacking or server crash. We presented a new strategy that is, Fully Homo morphic Encryption (FHE) algorithm for taking multiple copies of information. The FHE algorithm is used to enhance security to the information stored in the servers. When the data proprietor uploads the file, the FHE algorithm splits the file and takes multiple copies of the file. These copies are zipped in order to decrease memory size and are stored in multiple servers. The stored files are encrypted to provide maximum security. The FHE algorithm provides keygen, copygen and taggen, using these keys the data can be decrypted and retrieved.
\end{abstract}

Keywords- Multicloud storage, information leakage, system attackability, remote synchronization, distribution and optimization.

\section{INTRODUCTION}

\subsection{Motivation and Challenges}

$W_{\text {ith the inexorably fast take-up of gadgets, for }}$ example, workstations, mobiles and tablets, clients require an omnipresent. What's more, gigantic system stockpiling to deal with their regularly developing advanced lives. To satisfy these needs, many cloud-based capacity and document sharing administrations, for example, Dropbox, Google Drive and Amazon S3, have picked up fame due to the simple to- utilize interface and low stockpiling expense.

Nonetheless, these brought together

distributed storage administrations are scrutinized for getting the control of clients' information, which permits stockpiling suppliers to run investigation for promoting and publicizing. Moreover, the data in clients' information

Revised Manuscript Received on November 22, 2019

* Correspondence Author

M.Gowthami, Assistant Professor, Department of Computer Science and Engineering, Vel Tech High Tech Dr.Rangarajan Dr.Sakunthala Engineering College, Chennai, Mail: gowthamim@velhightech.com

Dr.S.Thanga Ramya, Associate Professor, Department of Computer Science and Engineering RMD Engineering College, Chennai,

Mail: str.it@rmd.ac.in can be spilled e.g., by methods of malevolent insiders, secondary passages, influence and intimidation. One conceivable answer to lessen the danger of data spillage is to utilize multi cloud capacity frame works ${ }^{[6],[16],[15],[17]}$ in which no single purpose of assault can release all the data.

A malignant element, for example, the one uncovered in ongoing assaults on protection ${ }^{[20]}$, would be required to pressure all the unique CSPs on which a client may put her information, so as to get a complete image of her information. Put essentially, as the colloquialism goes, attempt not to place all the investments knotted up on one residence.

However, the circumstance isn't so straightforward. CSPs, for example, Dropbox, among numerous others, utilize rsync-like conventions [2] to synchronize the nearby document to remote record in their incorporated mists ${ }^{[3]}$. Each nearby document is divided into little lumps also, these lumps are hashed with fingerprinting calculations for example, FHE, SHA-1, and MD5. Along these lines, a document's substance can be exceptionally recognized by this rundown of hashes. For each refresh of neighborhood document, just pieces with changed hashes will be transferred to the cloud. This synchronization dependent on hashes is extraordinary.

\section{RELATED WORK}

In this unit, we will review some of the literature related to the four distinct pillars of our work, which are as follows: Untrusted storage cloud: Depot ${ }^{[19]}$ and SPORC ${ }^{[4]}$ assumed that the storage clouds are untrusted and fault prone black boxes. However, both their work employed only a single cloud which has both compute and storing capacity. Our work is different since we consider a mutli cloud in which each storage cloud is only served as storage without the ability to compute. The earlier previous work such as Cooperative File System (CFS) [9] and Samsara ${ }^{[18]}$ designed their storage system with a peer-to-peer Network comprised of potentially untrusted nodes. Our work targets to use storage cloud without using decentralized P2P protocol [11] and optimizes data placement in a centralized way.

This paper extends our work on StoreSim ${ }^{[21]}$ Multicloud storage services. Our work is not alone in storing data with the adoption of multiple CSPs, e.g. SPANStore ${ }^{[17]}$ DepSky ${ }^{[6]}$ and NCCloud ${ }^{[16]}$. However, these work focused on different issues such as cost optimization [17], data consistency and 
Low Power Oriented Full Search Block Based Motion Estimation (LP-FSBME) Architecture Using Power Efficient Adder Compressor For H.265 Coding Techniques.

availability $^{[6]}$ and service response time ${ }^{[32]}$

Other efforts [12] on the cloud orchestration provided deployment plans in terms of the tradeoff between price and performance. Unlike these works, our effort focuses on the information leakage optimization for storage service in a multicloud environment by exploiting information similarity caused by the synchronization of modified data. Supplementary efforts on overcoming vendor lock-in, DepSky [6] minimized the cost of data transfer from one cloud to another by storing only a fraction of the total amount of data in each cloud while Scalia ${ }^{[15]}$ employed the data replication at a higher storage cost.

However, in StoreSim, we provide a user-specific weight for each cloud which not only coordinates the fraction of storage load for each cloud but also prevents the information leakage across the CSPs. Other studies have focused on measurement analysis of cloud storage services [3], [5]. Their work provided us with many insights on designing StoreSim. But their work failed to reveal optimization aspects of information leakages of the commercial CSPs they studied. Cloud security. Many studies ${ }^{[24],[22],[25]}$ focus on security and privacy aspects which are major obstacles of cloud adoption for both individuals and companies. Previous work ${ }^{[22]}$ proposed a semantic framework based on crowd-sourcing to determine the sensitivity of items and diverse attitudes of users towards privacy. Bohli et al. ${ }^{[24]}$ provided a survey for four different multi cloud architectures with various security and privacy- enhancing designs.

The architecture of StoreSim is one of them, which permits distributing fine-grained fragments of the data to different clouds. Our work further implements the StoreSim system with new information leakage measures.

Near-duplicate detection. Li et al. ${ }^{[23]}$ proposed a privacy loss measure based on the JS-divergence distance which is a technique of measuring the relationship between two probability distributions. Inspired by their work, we design our information leakage function based on similarity.

To compute the information leakage, we need to compute the pairwise similarities. MinHash[34], [35] and SimHash[35],[14] were designed for detecting the near-duplicate web pages based on Jaccard and Hamming distance, respectively. However, their work cannot apply to our work directly due to heavy computation and high storage overhead. To the best of our knowledge, this is the primary work which applies near-duplicate techniques for preventing information leakage in multi cloud storage services.

\section{PROPOSED PREDICTING MODULE}

\section{Cloud Service supplier:}

Cloud Service supplier gives administration to the client. Client registers the subtleties to specialist organization before sending the record. After client enrollment, administrators confirm the client profile and acknowledge the client.
Administrator might dismiss the unauthorized profile subtleties.

\section{Client transfer with keygen:}

After administrator confirms the client subtleties, User is now allowed to transfer. While transfer the document, records are scrambled and place away in the database and envelope. While transfer the information are splitted and stored in various server and so the programmer cannot hack the server information. Since information parts are place away in various server Key gen calculation are made and the information is transferred and scrambled.

\section{Document Conversion:}

While transferring information to server all information are in ZIP position to lessen the memory space occupied.

Approved clients:

Only the approved clients can request to the file administrator and the data owner.

Record ask:

Client might want to share the documents to another clients. Shared clients brings the record from document owner. Shared client sends the record demand to document owner and naturally same demand is send to administrator.

At that point Admin confirm the server and give the key to client by getting the key from document owner. After receiving the key the client can request and get the file.

\section{Record recuperation:}

On the off chance that client document might be degenerated or might get erased. So if client erase any record from server, user can recoup the erased documents from document separately. It is valuable to all cloud users if client can recoup the erased record, client login and get the erased documents

\section{Report Generation:}

Administrator produces the reports for number of clients register in the cloud. Furthermore, also set up the confirm and not check subtleties.

\section{Completely Homomorphic Encryption (FHE):}

The first completely homomorphic encryption plot, taking care of a focal open issue in cryptography. Such a preparation enables one to shape discretionary capacities more encoded information exclusive of the decoding key one can expertly process a reduced ciphertext that scrambles for any productively calculable capacity. This issue was presented by Rivets. Completely homomorphic encryption has various applications. For instance, it empowers confidential inquiries to a web index - the client presents a jumbled inquiry and the internet hunter processes a short encoded reply while never pleasing a gander at the query free. It furthermore empowers looking for on scrambled information - a client stores encoded records on a remote document server and can afterward have the server recover immediately minutes that (when decoded) complete some Boolean requirement, in spite of the fact that the server can't decode the documents separately. All the more 
extensively, completely homomorphic encryption improves the productivity of secure multiparty calculation. Our growth starts with a various degree homomorphic "boos trappable" encryption plot that workings when the competence is the strategy own unscrambling capacity. At that point show how, through recursive self-installing, boots trappable encryption give entirely homomorphic encryption. The enlargement makes consumption of complex issues on perfect cross section.

\section{PROPOSED ARCHITECTURE:}

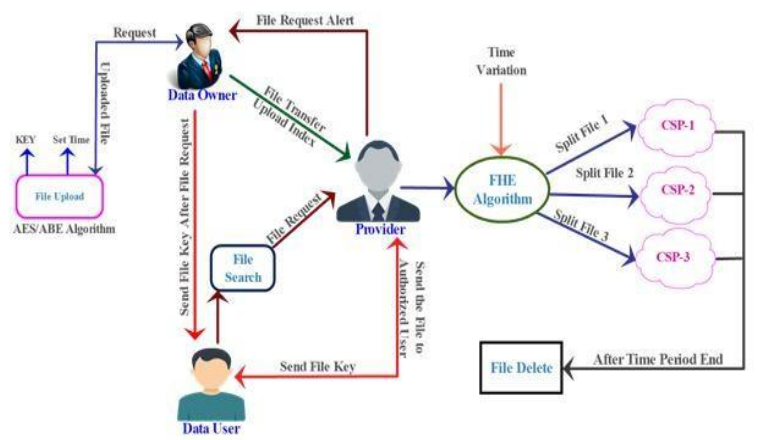

HOMOMORPHIC ENCRYPTION:

\section{Step 1: File transfer:}

The information[36] proprietor transfer the record to the cloud. The information proprietor will store their document in general society and private cloud.

\section{Stage 2: Encryption of the record}

The record is scrambled for the security safeguarding. The encoded record is put away in the cloud. The unapproved individuals can get to the document because of the encryption.

\section{Stage 3: Splitting the Documents}

The document is splitted and put away in the distinctive database.

\section{CONCLUSION}

We proposed a hybrid scheme that combines public key encryption and fully homomorphic encryption. The proposed scheme is suitable for cloud computing environments since it has low storage requirement, and supports efficient computing on encrypted data. Our solution provides the size of the transmitted ciphertexts and the conversion. The parameters of our hybrid scheme are very large when the message space of the FHE.

\section{REFERNCES}

[1] Hao Zhuang, Rameez Rahman, Pan Hui, Karl Aberer "Optimizing Information Leakage in Multicloud Storage Services" IEEE transactions on cloud computing, VOL.14,NO.8, AUG 2018.

[2] G. Greenwald and E. MacAskill, "Nsa prism program taps in to user data of apple, google and others," The Guardian, vol. 7, no. 6, pp. 1-43, 2013. IEEE TRANSACTIONS ON CLOUDCOMPUTING, VOL. 14, NO. 8, AUG 201814

[3] H. Zhuang, R. Rahman, P. Hui, and K. Aberer, "Storesim: Optimizing information leakage in multicloud storage services," in Cloud

Computing Technology and Science (CloudCom), 2015 IEEE 7th International Conference on. IEEE, 2015, pp. 379-386

[4] H. Zhuang, R. Rahman, and K. Aberer, "Decentralizing the cloud: How can small data centers cooperate?" in Peer-to-Peer Computing (P2P), 14-th IEEE International Conference on. Ieee, 2014, pp. 1-10.

[5] S. Choy, B. Wong, G. Simon, and C. Rosenberg, "A hybrid edge-cloud architecture for reducing on- demand gaming latency," Multimedia Systems, pp. 1-17,2014

[6] H. Harkous, R. Rahman, and K. Aberer, "C3p: Context-aware crowdsourced cloud privacy," in $14^{\text {th }}$ Privacy Enhancing Technologies Symposium (PETS 2014), 2014. H. Harkous, R. Rahman, and K. Aberer, "C3p: Context-aware crowdsourced cloud privacy," in 14th Privacy Enhancing Technologies Symposium (PETS 2014), 2014.

[7] A. Bessani, M. Correia, B. Quaresma, F. Andre, and P. Sousa, "Depsky: dependable and secure storage in a cloud-of-clouds," ACM Transactions on Storage (TOS), vol. 9, no. 4, p. 12, 2013.

[8] H. Chen, Y. Hu, P. Lee, and Y. Tang, "Nccloud: A network-coding-based storage system in a cloud-of- clouds," 2013.

[9] Z. Wu, M. Butkiewicz, D. Perkins, E. Katz- Bassett, and H. V. Madhyastha, "Spanstore: Cost- effective geo-replicated storage spanning multiple cloud services," in Proceedings of the Twenty-Fourth ACM Symposium on Operating Systems Principles. ACM, 2013, pp. 292-308.

[10] I. Drago, E. Bocchi, M. Mellia, H. Slatman, and A. Pras, "Benchmarking personal cloud storage," in Proceedings of the 2013 conference on Internet measurement conference. ACM, 2013, pp. 205-212.

[11] J.-M. Bohli, N. Gruschka, M. Jensen, L. L. Iacono, and N. Marnau, "Security and privacy-enhancing multicloud architectures," Dependable and Secure Computing, IEEE Transactions on, vol. 10, no. 4, pp. 212-224, 2013.

[12] T. G. Papaioannou, N. Bonvin, and K. Aberer, "Scalia: an adaptive scheme for efficient multi-cloud storage," in Proceedings of the International Conference on High Performance Computing, Networking, Storage and Analysis. IEEE Computer Society Press, 2012, p. 20.

[13] I. Drago, M. Mellia, M. M Munafo, A. Sperotto, R. Sadre, and A. Pras, "Inside dropbox: understanding personal cloud storage services," in Proceedings of the

2012 ACM conference on Internet measurement conference. ACM, 2012, pp. 481-494.

[14] T. Zou, R. Le Bras, M. V. Salles, A. Demers, and J. Gehrke, "Cloudia: a deployment advisor for public clouds," in Proceedings of the VLDB Endowment, vol. 6, no. 2. VLDB Endowment, 2012, pp. 121-132.

[15] P. Mahajan, S. Setty, S. Lee, A. Clement, L. Alvisi, M. Dahlin, and M. Walfish, "Depot: Cloud storage with minimal trust," ACM Transactions on Computer Systems (TOCS), vol. 29, no. 4, p.12,2011

[16] I. Ion, N. Sachdeva, P. Kumaraguru, and S. Capkun, "Home is safer than the cloud!: privacy concerns for consumer cloud storage," in Proceedings of the Seventh Symposium on Usable Privacy and Security. ACM, 2011, p. 13.

[17] A. Rajaraman and J. D. Ullman, Mining of massive datasets. Cambridge University Press, 2011.

[18] A. J. Feldman, W. P. Zeller, M. J. Freedman, and E. W. Felten, "Sporc: Group collaboration using untrusted cloud resources." in OSDI, vol. 10, 2010, pp.337-350.

[19] P. Li and C. Konig, "b-bit minwise hashing," in " Proceedings of the 19th international conference on World wide web. ACM, 2010, pp. 671-680.

[20] T. Li and N. Li, "On the tradeoff between privacy and utility in data publishing," in Proceedings of the

15th ACM SIGKDD international conference on Knowledge discovery and data mining. ACM, 2009, pp. 517-526.

[21] G. S. Manku, A. Jain, and A. Das Sarma, "Detecting near-duplicates for web crawling," in Proceedings of the 16th international conference on World Wide Web. ACM, 2007, pp. 141-150.

[22] M. Henzinger, "Finding near-duplicate web pages: a large-scale evaluation of algorithms," in Proceedings of the 29th annual international ACM SIGIR conference on Research and development in information retrieval. ACM, 2006, pp. 284-291.

[23] P. Berkhin, "A survey of clustering data mining techniques," in Grouping multidimensional data. Springer, 2006, pp. 25-71.

[24] A. Broder and M. Mitzenmacher, "Network applications of bloom filters: A survey," Internet mathematics, vol. 1, no. 4, pp. 485-509, 2004.

[25] L. P. Cox and B. D. Noble, "Samsara: Honor among thieves in peer-to-peer storage," ACM SIGOPS Operating Systems Review, vol. 37, no. 5, pp. 120-132, 2003. 
[26] M. S. Charikar, "Similarity estimation techniques from rounding algorithms," in Proceedings of the thiry-fourth annual ACM symposium on Theory of computing. ACM, 2002, pp. 380-388.

[27] T. Suel and N. Memon, "Algorithms for delta compression and remote file synchronization," 2002.

[28] F. Dabek, M. F. Kaashoek, D. Karger, R. Morris, and I. Stoica, "Wide-area cooperative storage with cfs," in ACM SIGOPS Operating Systems Review, vol. 35, no. 5. ACM, 2001, pp. 202-215.

[29] A. Z. Broder, S. C. Glassman, M. S. Manasse, and G. Zweig, "Syntactic clustering of the web," Computer Networks and ISDN Systems, vol. 29, no.

8, pp. 1157-1166, 1997

[30] U. Manber et al., "Finding similar files in a large file system." in Usenix Winter, vol. 94, 1994, pp. 1-10.

[31] J. W. Hunt and M. MacIlroy, An algorithm for differential file comparison. Bell Laboratories, 1976.

[32] "Prism surveillance program by nsa," http://en.wikipedia.org/wiki/ Edward Snowden\#Disclosure.

[33] "Emc hybrid cloud computing," http://www.emc.com/cloud/ hybrid-cloud- computing/index.htm.

[34] "Ibm multicloud toolkit,"

http://www.zurich.ibm.com/csc/security/ toolkit/.

[35] "Microsoft hybrid clouds," http://www.microsoft.com/en-us/ servercloud/solutions/hybrid-cloud.aspx.

[36] "Novel Effective X-Path Particle Swarm Extraction based Retrieval for Deprived Videos", Springer Cluster computing, Oct 2017.

\section{AUTHORS PROFILE}

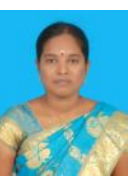

Mrs. M. Gowthami completed U.G, B.E (CSE) in IFET College of Engineering, with First Class in 2012 and M.E from Agni College of Technology, with First Class with Distinction in 2014.She is an Assistant Professor in the department of CSE and has a teaching experience of 5 years. She is currently pursuing her Ph.D.,(ICE) in Anna University, Chennai. Her research interests include, Wireless Network and Network Security.

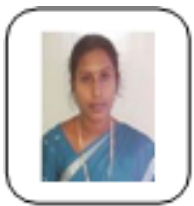

Dr. S. Thanga Ramya, B.E, M.S (by Res), Ph.D, is an Associate Professor in the Department of Information Technology, since June 2008. She obtained her B.E. (CSE) from Dr.Sivanthi Aditanar College of Engineering and M.S by Research (ICE) from Anna University, Chennai. She has obtained her Ph.D in Information and Communication Engineering from Anna University, Chennai, in 2017. She has been in the teaching profession for the past 18 years and has handled both UG and PG programs. Her areas of interest include programming languages, database management and data mining. She has published 9 papers in various International Journals and Conferences. She has attended many workshops \& FDPs sponsored by AICTE related to her area of interest. She has also published 4 books. She is the life member of ISTE. 